\title{
Recovering directed networks in neuroimaging datasets using partially conditioned Granger causality
}

$\mathrm{G} \mathrm{Wu}^{1,2^{*}}$, W Liao ${ }^{3}, \mathrm{~S}$ Stramaglia ${ }^{4}, \mathrm{D}$ Marinazzo ${ }^{1 *}$

From Twenty Second Annual Computational Neuroscience Meeting: CNS*2013

Paris, France. 13-18 July 2013

We address the computational and conceptual problems arising when conditional Granger causality, necessary to disambiguate direct and mediated influences, is used on short and noisy datasets of many variables, as it is typically the case in some EEG protocols and in fMRI. We show that considering Granger causality in the framework of information theory we can limit the conditioning to a limited number of variables chosen as the most informative, obtaining more stable and reliable results on fMRI datasets both at region and voxel level.

The results on a dynamical model simulated on the structural connectome matrix show that PCGC performs
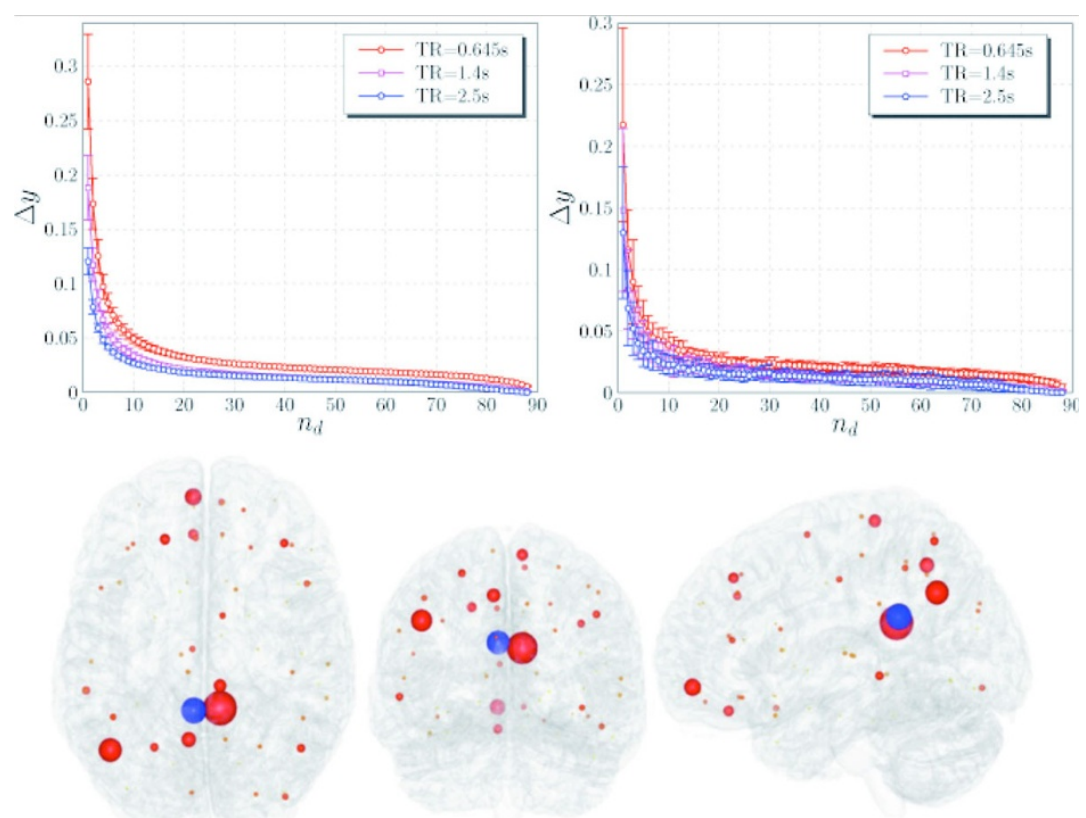

Figure 1 Top: the residual information gain $\Delta y$ when an additional variable nd is added to the conditioning process, averaged over all targets (left), and for a specific target, PCG.L (right). Bottom: the distributions of the 10 most informative regions for PCG.L

\footnotetext{
* Correspondence: daniele.marinazzo@gmail.com

${ }^{1}$ Faculty of Psych. and Ed. Sciences, Dept. of Data Analysis, Ghent University,

1, B-9000 Ghent, Belgium

Full list of author information is available at the end of the article
} 
significantly better than fully conditioned and pairwise $\mathrm{GC}$, in particular when the signals are short.

We then apply PCGC to publicly available fMRI datasets, with different TRs and divided in two sessions. It is shown that the series with shorter TR have highest informational content. The curve of the mutual information gain when an additional variable is used for conditioning starts to become flat around 10 variables, indicating the optimal number of variables to condition to. These variables are located not only in proximity of the target variable, but distributed across what we could call an "informational resting state network" across the whole brain. This behavior is stable across sections and TRs.

With the additional step of pre-selecting the variables according to the community structure, PCGC is applied as well to time series from individual voxels. This allows to extend the concept of functional connectivity density to effective connectivity, revealing hubs for incoming and outgoing information transfer.

\section{Conclusions}

Apart from solving the computational and conceptual issues arising from a full conditioning of GC, this approach presents a new way of looking at neuroimaging datasets, using the concept of informative clustering to group the variables from different brain regions in terms of their shared information on the future of another target variable.

\section{Author details}

${ }^{1}$ Faculty of Psych. and Ed. Sciences, Dept. of Data Analysis, Ghent University, 1, B-9000 Ghent, Belgium. ${ }^{2}$ Key Laboratory for Neurolnformation of Ministry of Education, UESTC, Chengdu, 610054, China. ${ }^{3}$ Center for Cognition and Brain Disorders, Hangzhou Normal University, Hangzhou 310015, China. ${ }^{4}$ Dipartimento di Fisica e Istituto Nazionale di Fisica Nucleare, Sezione di Bari, Bari, 70126, Italy.

Published: 8 July 2013

\section{References}

1. Deshpande G, LaConte S, James GA, Peltier S, Hu X: Multivariate Granger causality analysis of fMRI data. Hum Brain Mapp 2008, 30:1361-1373.

2. Marinazzo D, Pellicoro M, Stramaglia S: Causal information approach to partial conditioning in multivariate data sets. Comput Math Methods Med 2012, ID303601.

3. Tomasi D, Volkow N: Functional connectivity density mapping. Proceedings of the National Academy of Sciences 2010, 107:9885-9890.

\section{Submit your next manuscript to BioMed Central} and take full advantage of:

- Convenient online submission

- Thorough peer review

- No space constraints or color figure charges

- Immediate publication on acceptance

- Inclusion in PubMed, CAS, Scopus and Google Scholar

- Research which is freely available for redistribution

Submit your manuscript at www.biomedcentral.com/submit
C Biomed Central 